EPJ Web of Conferences 112, 03001 (2016)

DOI: 10.1051 /epiconf/201611203001

(C) Owned by the authors, published by EDP Sciences, 2016

\title{
Tagged spectator deep-inelastic scattering off the deuteron as a tool to study neutron structure
}

\author{
Wim Cosyn ${ }^{1, a}$ and Misak Sargsian ${ }^{2, b}$ \\ ${ }^{1}$ Department of Physics and Astronomy, Proeftuinstraat 86, Ghent University, 9000 Ghent, Belgium \\ ${ }^{2}$ Department of Physics, Florida International University, Miami FL 33199, U.S.A.
}

\begin{abstract}
We give an overview of a model to describe deep-inelastic scattering (DIS) off the deuteron with a spectator proton, based on the virtual nucleon approximation (VNA). The model accounts for the final-state interactions (FSI) of the DIS debris with the spectator proton. Values of the rescattering cross section are obtained by fits to high-momentum spectator data. By using the so-called "pole extrapolation" method, free neutron structure functions can be obtained by extrapolating low-momentum spectator proton data to the on-shell neutron pole. We apply this method to the BONuS data set and find a surprising Bjorken $x$ dependence, indicating a possible rise of the neutron to proton structure function ratio at high $x$.
\end{abstract}

\section{Introduction}

Tagged proton spectator DIS off the deuteron $\left(e+d \rightarrow e^{\prime}+X+p_{s}\right)$ can be used in several ways to study the role of QCD dynamics at nucleonic length scales. At low spectator momenta, one has access to the structure functions of an almost on-shell neutron. Together with proton structure functions, flavor separation can be performed and the $d$ and $u$ quark densities can be determined. At higher proton spectator momenta, the deuteron is in a high-density configuration and medium modifications of the structure functions can be quantified. In kinematics where FSI mechanisms are large, one can use the reaction to constrain models for the $X$-proton rescattering. Recently, two experiments at Jefferson Lab have measured the reaction in different kinematics: Deeps at higher spectator momenta of a few hundred $\mathrm{MeV}$ [1] and, BONuS at lower ones around $100 \mathrm{MeV}$ [2]. In the near future, the BONuS12 experiment will run [3] and the reaction is considered as a method to extract neutron structure at a future electron-ion collider [4]. In order to provide meaningful interpretations of the measured data, theoretical models that quantify the importance of the FSI in the reaction are needed [5-10]. The major difficulty in doing this is that one lacks detailed information about the composition and spacetime evolution of the hadronic system produced in the deep-inelastic scattering and how this changes as a function of Bjorken $x$ and $Q^{2}$. Here, we present work in a model that has been used to account for FSI mechanisms at moderate and high Bjorken $x$ in $e+d \rightarrow e^{\prime}+X+p_{s}$ [11-13] as well as inclusive deuteron DIS [14, 15].

\footnotetext{
a e-mail: wim.cosyn@ugent.be

be-mail: sargsian@fiu.edu
} 


\section{Formalism}

The model introduced in Ref. [11] accounted for FSI effects in DIS from the deuteron based on general properties of high-energy diffractive scattering. The underlying assumption was that due to the restricted phase space (finite values of $W$ and $Q^{2}$ ), the minimal Fock state component of the wave function can be used to describe DIS from the bound nucleon. In this case the scattered state consists of three outgoing valence quarks. The model uses the virtual nucleon approximation (VNA) $[6,7,16]$ and only considers the proton-neutron component of the deuteron wave function. The effects of FSI are encoded in an effective rescattering amplitude between the produced hadronic mass $X$ and the spectator proton. Given the $\mathrm{GeV}$ range of the energies in the experiments, the rescatterings will occur over small angles and be highly diffractive. This allows us to model the rescattering amplitude using the generalized eikonal approximation (GEA) [17]. In the GEA, the eikonal rescattering amplitude $f_{X^{\prime} N, X N}$ is parametrized using three parameters (total rescattering cross section $\sigma_{X N}$, slope factor $B$, and real part $\epsilon$ ), that have a dependence on the $Q^{2}$ and Bjorken $x$ (or equivalently invariant mass $W$ of hadronic products $X$ ):

$$
f_{X^{\prime} N, X N}=\sigma\left(Q^{2}, x\right)\left[i+\epsilon\left(Q^{2}, x\right)\right] e^{\frac{B\left(Q^{2}, x\right)}{2} t} .
$$

The cross sections including FSI are obtained in a factorized approach, whereby the DIS interaction of the virtual photon with the off-shell nucleon (encoded in the structure functions $F_{i N}$ ) is taken out of the integration over the momentum of the on-shell intermediate spectator nucleon. Finally, the four structure functions of the tagged spectator DIS process can be written in the following form

$$
F_{i}^{S I}=\left(\alpha(i) F_{1 N}+\beta(i) F_{2 N}\right) S^{D}\left(p_{s}\right)(2 \pi)^{3} 2 E_{s}, \quad \text { with } i=\text { L,T,LT,TT. }
$$

Here, $\alpha(i), \beta(i)$ are kinematical factors (see Ref. [11] for detailed expressions), and $S^{D}\left(p_{s}\right)$ is the distorted deuteron momentum distribution that contains an impulse approximation (IA) contribution and an FSI contribution including the rescattering amplitude of Eq. (1) [11].

Even for very low spectator momenta, the struck neutron is still off-shell in the deuteron. One can still perform an analytical continuation, however, of the amplitude into the unphysical kinematical region and extrapolate it to the on-shell point $p_{i}^{2}=\left(p_{D}-p_{s}\right)^{2}=m_{n}^{2}$. The IA part of the amplitude has a pole at this value of $p_{i}^{2}$, while the FSI part does not due to the additional integration over the loop momentum. This is the so-called "no-loop theorem" [7]. This means carrying out the pole extrapolation automatically gets rid of nuclear effects and allows one to extract the on-shell neutron structure function. This procedure is similar to the ones first proposed by Chew and Lowin hadronproton scattering [18]. Due to the small binding energy of the deuteron, the actual extrapolation length to the on-shell pole is very small, and there are also no issues regarding a zero crossing for $p_{i}^{2}$. The pole extrapolation procedure for the extraction of $F_{2 n}$ consists of multiplying the measured deuteron structure function, $F_{2 D}^{S I \text {,EXP }}$ by the factor $I\left(\alpha_{s}, \boldsymbol{p}_{s \perp}, t\right)$ [7], which cancels the singularity of the IA amplitude and is normalized such that

$$
F_{2 n}^{\mathrm{extr}}\left(Q^{2}, x, p_{i}^{2}\right)=I\left(\alpha_{s}, \boldsymbol{p}_{s \perp}, p_{i}^{2}\right) \cdot F_{2 D}^{S I, \mathrm{EXP}}\left(Q^{2}, x, \alpha_{s}, \boldsymbol{p}_{s \perp}\right)
$$

approaches the free $F_{2 n}\left(Q^{2}, x\right)$ in the $p_{i}^{2} \rightarrow m_{n}^{2}$ limit with FSI effects being diminished.

\section{Results}

Figure 1 shows a comparison between one data set from the high spectator momentum deeps experiment [1] with our model calculations. The most striking feature of the data is the large excess at forward spectator angles. This is markedly different from the quasi-elastic breakup case where the 

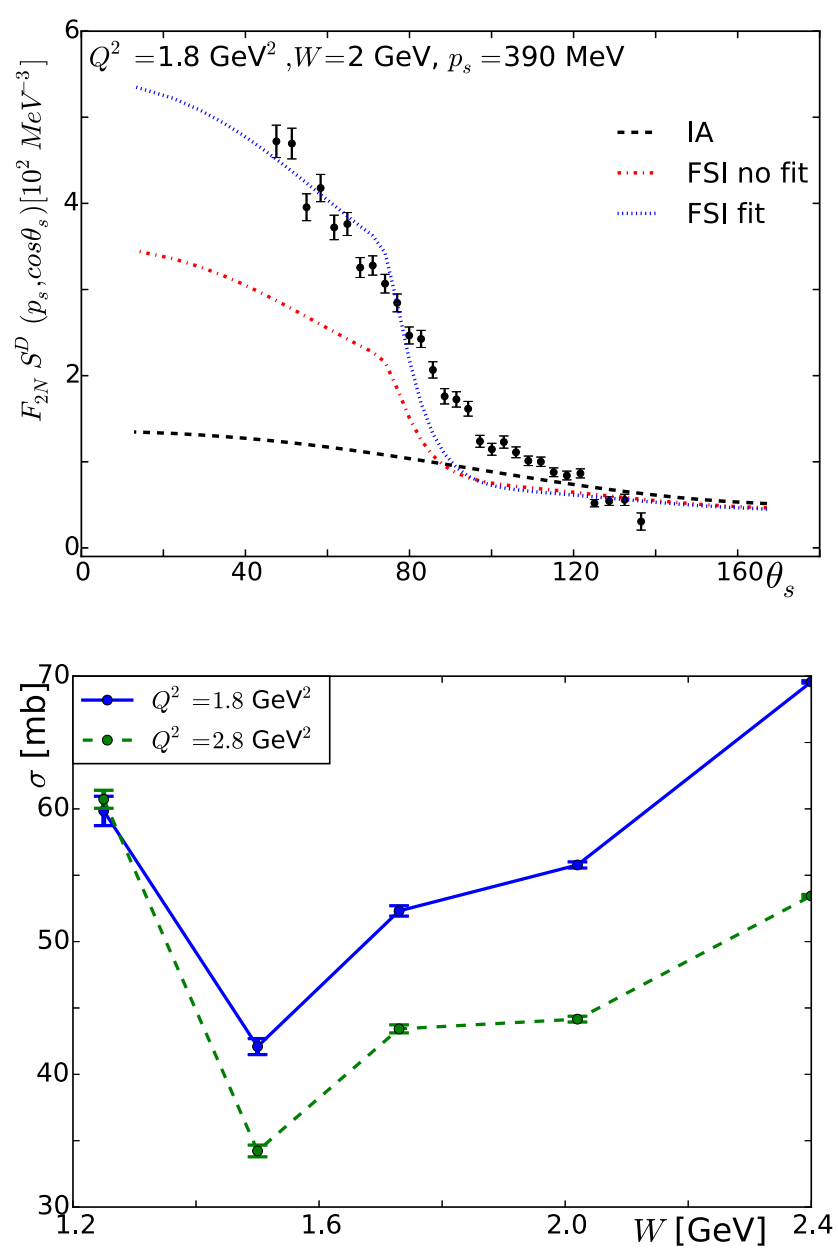

Figure 1. Comparison between deeps data [1], the plane-wave IA calculation (dashed black curve), an unfitted FSI calculation using standard $N N$ scattering values of $\sigma=40 \mathrm{mb}, B=8 \mathrm{GeV}^{-2}$, $\epsilon=-0.5$ (red dash-dotted), and a FSI calculation where $\sigma=55.8$ was mb was fitted (blue dotted).

Figure 2. Values of the fitted $\sigma$ rescattering parameter in Eq. (1) for $Q^{2}=1.8 \mathrm{GeV}^{2}$ (dashed green curve) and $Q^{2}=2.8 \mathrm{GeV}^{2}$ (full blue curve), obtained by fitting the high momentum spectator deeps data.

cross section peaks in the perpendicular region [19]. It is clear the IA calculation has an almost flat dependence on the spectator angle and can in no way reproduce the full range of the data. An FSI calculation with values of the rescattering parameters inspired by the known $N N$ ones is able to reproduce the angular dependence better but still underestimates the data. Intuitively, this is no surprise as for the value of $W=2 \mathrm{GeV}$ in Fig. 1, we are well above the nucleon mass. Therefore, we have fitted a value of $\sigma$ for each $\left\{W, Q^{2}\right\}$ setting of the data. The calculation using this fitted parameter is also shown for the kinematics of Fig. 1. For a comparison with the full deeps data set, we refer to Ref. [11].

In Fig. 2, we show the $W$ and $Q^{2}$ dependence of the fitted values of $\sigma$ from our analysis of the deeps data. The parameter $\sigma$ has a value of about $60 \mathrm{mb}$ at the $\Delta$-resonance, and then clearly increases with increasing invariant mass $W$ of the hadronic products. More interesting is the dependence on $Q^{2}$, where there is a significant decrease in the $\sigma$ parameter with increasing $Q^{2}$. This could be a sign of a color transparency signal in this reaction, where the hadronic products are produced in a colorless point-like configuration at higher momentum transfers, which experiences reduced strong interactions with the remaining hadrons. More measurements at higher $Q^{2}$ values are needed, however, to further investigate this behavior. 


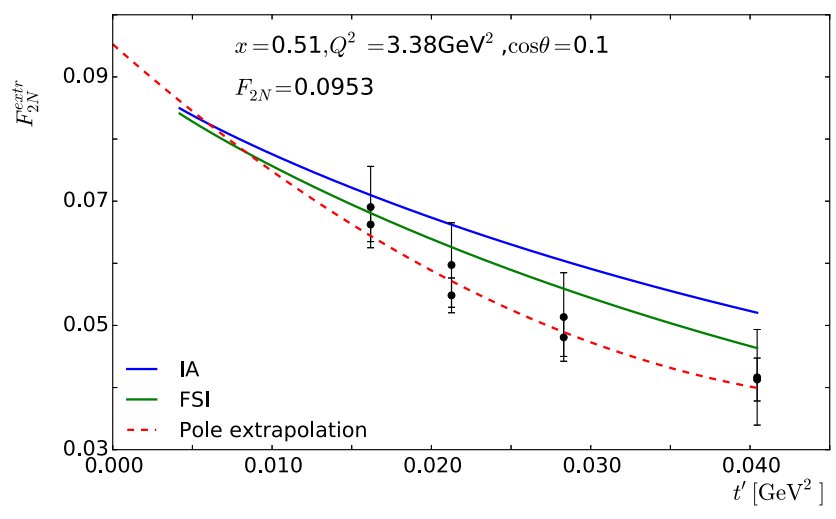

Figure 3. Example of the pole extrapolation method using the renormalized BONuS data (black circles) with the quadratic pole extrapolation curve (red dashed curve) as a function of $t^{\prime 2}=p_{i}^{2}-m_{n}^{2}$. The IA (full blue curve) and FSI (full green curve) calculations are shown for comparison.

In Ref. [12], the pole extrapolation method was applied to the high momentum spectator deeps data. Given the large extrapolation length, no robust results were obtained for $F_{2 n}$. With the more recent low spectator momentum BONuS data, which measured down to proton momenta of $70 \mathrm{MeV}$, the extrapolation length is very short and pole extrapolation became applicable. One problem with the BONuS data is that the measurements for different spectator momenta happened at different and not well known efficiencies. In the original analysis, the data was therefore normalized to an IA model [20] in the backward spectator region. In Ref. [13], we renormalized the BONuS data using our FSI model at high $W, Q^{2}$ (where the neutron structure function is quite well known), using the rescattering parameter values extracted in our previous fit to the the deeps data. Taking these renormalized data, we implemented the pole extrapolation method for the BONuS data. Figure 3 shows an example for one kinematical setting. The two data points at each $t^{\prime}$ value correspond to data taken at two initial beam energy values and one can clearly observe they are consistent with each other. Plotting our model calculations with the data, it is also clear that through implementing Eq. (3), FSI effects indeed become smaller as one moves closer to the on-shell pole. As the extrapolation curve shows in Fig. 3, the extrapolation distance to the on-shell point is reasonable.

For each kinematical setting of the BONuS data, pole extrapolation was carried out as in Fig. 3, and a weighted average was taken over all measured spectator angle bins. The final result is shown in Fig. 4 as the ratio of $F_{2 n} / F_{2 p}$ where the parametrization of Ref. [21] was used for the $F_{2 p}$ values. We repeat that the values obtained in this manner are free of Fermi motion and nuclear medium modification effects. The systematic errors were estimated by taking all uncertainties in the data and our normalization procedure (deuteron wave function, structure function parametrization, renormalization fit) into account in a Monte Carlo simulation [13]. The most striking feature in Fig. 4 is the rise of the ratio at large Bjorken $x$. The results at lower Bjorken $x<0.5$ are in agreement with existing estimates. It is worth noting that the upward trend of the ratio at higher $x$ is not due to our renormalization of the data. Indeed, the values of the ratio are higher without the renormalization applied. As the $W$ $(\approx 1.18 \mathrm{GeV}$ ) values corresponding to the highest $x$ values in Fig. 4 are in the sub-DIS regime, one cannot directly relate the rise of the ratio to the underlying properties of the $u$ and $d$ quark pdf's. Such a connection can only be made using duality arguments. It is worth noting, however, that the duality analysis of the BONuS data [22], concluded that the $\Delta$-resonance contributes to the duality, within 20$30 \%$ accuracy. One possible explanation of the rise could be the presence of isosinglet $q q$ short-range correlations (SRCs) in the nucleon at $x \rightarrow 1$. Such a correlation will result in the same momentum sharing effect, which is observed recently in asymmetric nuclei in the NN SRC region [23]. 


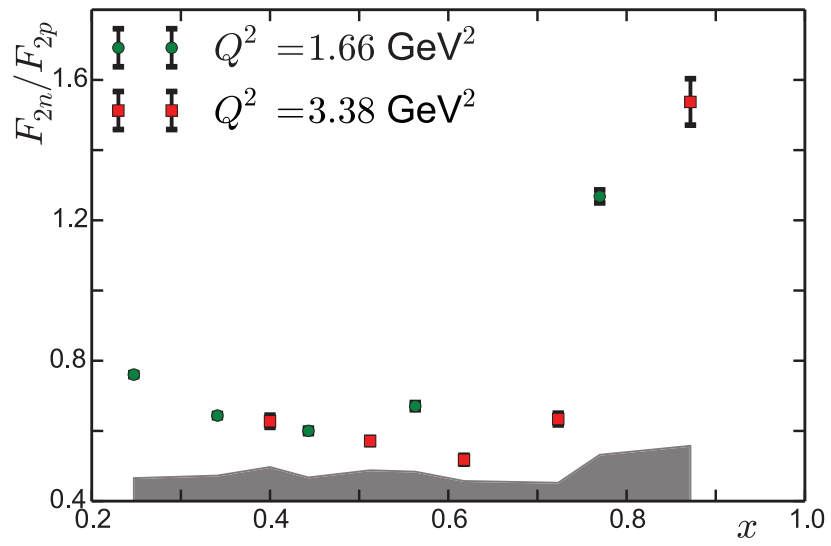

Figure 4. $F_{2 n}$ to $F_{2 p}$ ratio obtained using the pole extrapolation applied to the renormalized BONuS data. The grey band denotes estimated systematic errors in the procedure.

\section{Conclusion}

We have presented a selection of results obtained in a model used to describe the tagged spectator DIS process off the deuteron including the effect of FSI. We obtained a good description of data at higher spectator momenta and used these data to fit the $Q^{2}$ - and $W$-dependence of the rescattering parameter $\sigma$. For data at lower spectator momenta, we applied the pole extrapolation method, which allows to extract the free neutron structure function in a way that eliminates nuclear effects. We observe an intriguing rise of the $F_{2 n} / F_{2 p}$ ratio at high Bjorken $x$.

\section{Acknowledgements}

The computational resources (Stevin Supercomputer Infrastructure) and services used in this work were provided by Ghent University, the Hercules Foundation and the Flemish Government - department EWI. This work is supported by the Research Foundation Flanders as well as by the U.S. Department of Energy Grant under Contract DE-FG02-01ER41172.

\section{References}

[1] A.V. Klimenko et al. (CLAS), Phys. Rev. C73, 035212 (2006), nucl-ex/0510032

[2] N. Baillie, S. Tkachenko, J. Zhang, P. Bosted, S. Bültmann, M.E. Christy, H. Fenker, K.A. Griffioen, C.E. Keppel, S.E. Kuhn et al. (CLAS Collaboration), Phys.Rev.Lett. 108, 199902 (2012), 1110.2770

[3] S. Bueltmann (2006), jLAB-PR12-06-113

[4] W. Cosyn, V. Guzey, D.W. Higinbotham, C. Hyde, S. Kuhn, P. Nadel-Turonski, K. Park, M. Sargsian, M. Strikman, C. Weiss, J. Phys. Conf. Ser. 543, 012007 (2014), 1409. 5768

[5] S. Simula, Phys. Lett. B387, 245 (1996), nucl-th/9605024

[6] W. Melnitchouk, M. Sargsian, M.I. Strikman, Z. Phys. A359, 99 (1997), nucl-th/9609048

[7] M. Sargsian, M. Strikman, Phys. Lett. B639, 223 (2006), hep-ph/0511054

[8] C. Ciofi degli Atti, L.P. Kaptari, S. Scopetta, Eur. Phys. J. A5, 191 (1999), hep-ph/9904486

[9] C. Ciofi degli Atti, B.Z. Kopeliovich, Eur. Phys. J. A17, 133 (2003), nucl-th/0207001

[10] V. Palli, C. Ciofi degli Atti, L.P. Kaptari, C.B. Mezzetti, M. Alvioli, Phys. Rev. C80, 054610 (2009), 0911. 1377 
[11] W. Cosyn, M. Sargsian, Phys. Rev. C84, 014601 (2011), 1012.0293

[12] W. Cosyn, M. Sargsian, AIP Conf.Proc. 1369, 121 (2011), 1101. 1258

[13] W. Cosyn, M.M. Sargsian (2015), 1506.01067

[14] W. Cosyn, W. Melnitchouk, M. Sargsian, Phys.Rev. C89, 014612 (2014), 1311 . 3550

[15] W. Cosyn, M. Sargsian, J. Phys. Conf. Ser. 543, 012006 (2014), 1407. 1653

[16] M.M. Sargsian, S. Simula, M.I. Strikman, Phys. Rev. C66, 024001 (2002), nucl-th/0105052

[17] L.L. Frankfurt, M.M. Sargsian, M.I. Strikman, Phys. Rev. C56, 1124 (1997), nucl-th/9603018

[18] G.F. Chew, F.E. Low, Phys. Rev. 113, 1640 (1959)

[19] M.M. Sargsian, Phys. Rev. C82, 014612 (2010), 0910. 2016

[20] S. Tkachenko et al. (CLAS), Phys. Rev. C89, 045206 (2014), [Addendum: Phys. Rev.C90,059901(2014)], 1402.2477

[21] M. Christy, P.E. Bosted, Phys.Rev. C81, 055213 (2010), 0712. 3731

[22] I. Niculescu et al., Phys. Rev. C91, 055206 (2015), 1501.02203

[23] M.M. Sargsian, Phys. Rev. C89, 034305 (2014), 1210.3280 